\title{
IL-17-driven intestinal fibrosis is inhibited by Itch-mediated ubiquitination of HIC-5
}

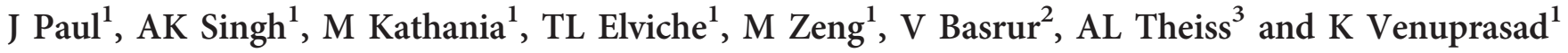

Intestinal fibrosis is a major complication in inflammatory bowel diseases, but the regulatory mechanism that inhibits fibrosis remains unclear. Here we demonstrate that Itch $^{-1-}$ myofibroblasts express increased amounts of profibrotic collagen type I and $\alpha$-SMA in response to IL-17. Mechanistically, we demonstrate that Itch directly binds to HIC-5 and targets it for K63-linked ubiquitination to inhibit IL-17-driven intestinal fibrosis. Reconstitution of Itch ${ }^{-1-}$ myofibroblasts with wild-type Itch but not the Itch-C830A mutant normalized the expression of profibrotic genes. Similarly, shRNA-mediated inhibition of HIC-5 normalized the expression of profibrotic gene expression. Thus, we have uncovered a novel mechanism by which Itch negatively regulates intestinal fibrosis.

\section{INTRODUCTION}

Inflammatory bowel diseases (IBDs) such as ulcerative colitis (UC) and Crohn's disease are chronic inflammatory disorders of the gastrointestinal tract that affect about 1.6 million Americans. ${ }^{1}$ Intestinal fibrosis is a common complication in IBDs that results in progressive tissue architectural distortion, loss of function, and luminal narrowing, which requires surgery. ${ }^{2}$ Fibrosis is caused by the deposition of extracellular matrix (ECM) proteins, including collagen and fibronectin, which are produced by the intestinal myofibroblasts in response to chronic inflammation. ${ }^{3}$

IL-17 produced by Th17 cells and innate lymphoid cells plays a crucial role in the pathogenesis of IBD. ${ }^{4-6}$ Development of Th17 cells requires the combined action of IL- 6 , TGF- $\beta$, IL- $1 \beta$, and IL-23. ${ }^{7}$ These cytokines, which are produced by the myeloid cells, promote the expression of the lineage-specific transcription factor ROR- $\gamma \mathrm{t}$, which is essential for IL-17 expression. ${ }^{7,8}$ Although IL-17 is linked to pulmonary, liver, and intestinal fibrosis, ${ }^{9}$ the mechanism by which IL-17 promotes fibrosis remains unclear.

Hydrogen peroxide-inducible clone 5 (HIC-5) is a member of the paxillin family protein that was originally identified as a TGF- $\beta 1$ and $\mathrm{H}_{2} \mathrm{O}_{2}$-inducible gene. ${ }^{10}$ The $\mathrm{N}$-terminal region of HIC-5 is composed of four LD domains, which are rich in Leu and Asp. The C-terminal region is composed of four LIM domains with two zinc fingers. ${ }^{11}$ Since both the LD and LIM domains are protein-protein interacting domains, HIC-5 acts as an adaptor and as a nuclear receptor coactivator. ${ }^{12,13}$ HIC-5 plays a crucial role in fibrosis by regulating differentiation of myofibroblasts and expression of ECM proteins. ${ }^{14-17}$

Ubiquitin-mediated post-translational modification regulates a variety of signaling pathways. ${ }^{18}$ Ubiquitination involves a cascade of biochemical reactions through ubiquitin activating (E1) enzymes, ubiquitin-conjugating (E2) enzymes, and ubiquitin ligase (E3) enzymes. ${ }^{18}$ The E3 ubiquitin ligases are critical components of this system because they recognize, bind to, and recruit specific target proteins for ubiquitination. ${ }^{18}$

Itch is an E3 ubiquitin ligase that belongs to the HECT (homologous to the E6-AP C terminus) family. Itch contains a protein kinase C-related C2 domain, four WW domains each of which contains two conserved tryptophan residues and the HECT ligase domain. ${ }^{19}$ The WW domains recognize the proline-rich Pro-Pro-X-Tyr (PPXY) consensus sequence (where $X$ is any amino acid) in their substrate targets. ${ }^{19}$ Itch deficiency in mice leads to multiorgan inflammatory disorders. ${ }^{19}$ Similarly, a truncated mutation of human Itch results in inflammatory disorders, including enteropathy. ${ }^{20}$ Here we demonstrate that Itch inhibits IL-17-driven fibrosis by targeting HIC-5 for ubiquitination. These findings could lead to advancement of therapeutic strategies for reducing fibrosis in IBD, which remains an unresolved clinical challenge.

${ }^{1}$ Baylor Institute for Immunology Research, Baylor Research Institute, Dallas, Texas, USA. ²Department of Pathology, University of Michigan, Ann Arbor, Michigan, USA and ${ }^{3}$ Department of Internal Medicine, Division of Gastroenterology, Baylor Research Institute, Baylor University Medical Center, Dallas, Texas, USA. Correspondence: K Venuprasad (venuprasad.poojary@BSWhealth.org)

Received 30 January 2017; accepted 14 May 2017; published online 14 June 2017. doi:10.1038/mi.2017.53 


\section{RESULTS}

\section{Itch deficiency leads to spontaneous colonic fibrosis}

Recently, we demonstrated that Itch-deficient mice develop spontaneous rectal prolapse due to severe colonic inflammation. ${ }^{21}$ Histological examination of Masson's trichrome-stained sections of colon tissues showed increased collagen fibers in the 6-8 month-old colon tissues of Itch $^{-1-}$ mice compared to agematched control mice (Figure 1a and b). To investigate if fibrosis and inflammation coexist, we performed hematoxylin and eosin staining, and Masson's trichrome staining in the same sections. As shown clearly in Supplementary Figure 1a and $\mathbf{1 b}$ online, we observed inflammation and fibrosis in adjacent areas. Since fibrosis is caused by pathological deposition of ECM proteins (collagens I-VI, $\alpha$-SMA, etc), we analyzed the expressions of collagen I (Col I) and $\alpha$-SMA by real-time polymerase chain reaction (PCR). As shown in Figure 1c and $\mathbf{d}$, we found a marked increase in the expression of these fibrosis-associated genes in the $\mathrm{Itch}^{-/-}$colon compared to the Itch ${ }^{+/+}$colon.

\section{Itch directly interacts with HIC-5}

Next, we sought to identify the dysregulated signaling pathways that lead to fibrosis in Itch-deficient mice using a proteomics approach. We performed pull-down assays using either glutathione S-transferase (GST) alone or a fusion protein of GST and Itch (GST-Itch) with the lysate of colon tissue from Itch $^{-1-}$ mice (Figure 2a). The precipitated proteins were resolved by $10 \%$ sodium dodecyl sulfate polyacrylamide gel electrophoresis (SDS-PAGE) and subjected to mass spectrometry (MS) analysis following in-gel digestion with trypsin. By this approach, we identified several potential Itch binding proteins in precipitates of GST-Itch. Since the WW domains of Itch bind to the proline-rich PPXY motifs on its target proteins, we short-listed the PPXY motif-containing proteins from the list of proteins identified in the MS analysis (Figure 2b). HIC-5 was one of the potential Itch-interacting proteins that contained the PPXY motif. A representative MS/MS spectrum corresponding to ${ }^{170}$ VQNHLPASGPPQPPAASPTR ${ }^{189}$ of the HIC-5 peptide sequence is shown in Figure 3a.

Since HIC-5 was shown to be involved in myofibroblast differentiation and fibrosis, ${ }^{14,16,22-24}$ we investigated if Itchmediated ubiquitination regulates colonic fibrosis. For this, we transiently transfected 293T cells with Flag-tagged HIC-5 (Flag-HIC-5) and Myc-tagged Itch (Myc-Itch). We immunoprecipitated proteins from cell lysates with control mouse immunoglobulin G (IgG), antibody to Flag (anti-Flag), or antibody to Myc (anti-Myc). Anti-Myc immunoprecipitated Flag-HIC-5 and anti-Flag immunoprecipitated Myc-Itch, which suggests that Itch interacts with HIC-5 (Figure 3b). Furthermore, we performed a pull-down assay using the lysates of 293T cells transfected with Flag-HIC-5 and bacterially expressed purified GST-Itch. GST-Itch but not GST alone precipitated Flag-HIC-5 (Figure 3c), confirming the Itch/ HIC-5 interaction. To determine whether this interaction occurs endogenously in the colonic tissue, we performed co-immunoprecipitation experiments with mouse embryonic fibroblasts (MEFs) from Itch ${ }^{+/+}$mice. We found that Itch co-immunoprecipitated with HIC-5 and vice versa (Figure 3d). To test if this interaction occurs through WW-PPXY motifs, we generated a HIC-5 mutant (Flag- $\Delta \mathrm{HIC}-5)$ in which the PPXY motif was deleted. We then transiently transfected 293T cells with Flag-HIC-5, Flag- $\Delta \mathrm{HIC}-5$ and Myc-Itch, and immunoprecipitated the cell lysates with anti-Flag. The immunoprecipitates were blotted with anti-Myc and then a
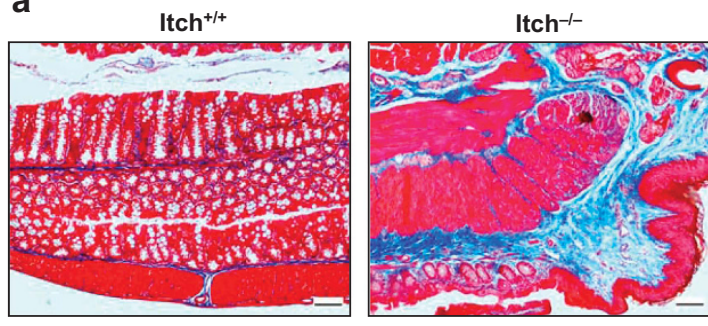

C

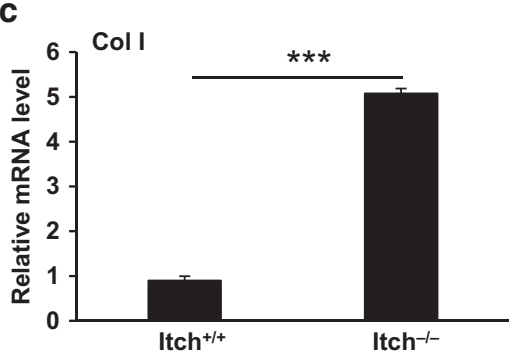

b

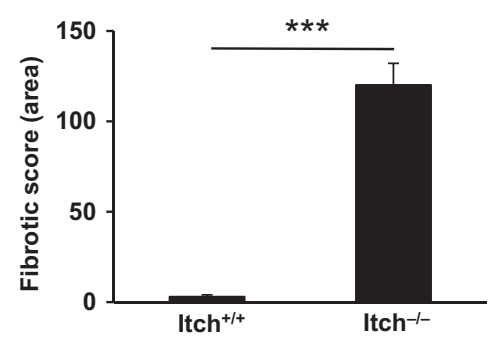

d

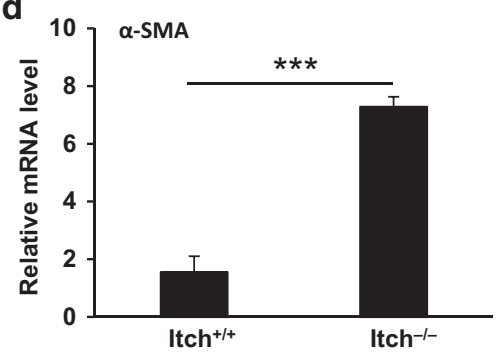

Figure 1 Severe fibrosis exists in the colon of Itch ${ }^{-/-}$mice. (a) Masson trichrome staining of collagen deposition in tissue sections of old ltch ${ }^{+/+}$and Itch ${ }^{-1-}$ mouse colons. Collagen was stained blue against a red background. (b) Fibrotic scores for sections in part a. (c, d) Relative mRNA levels of (c) Col I and (d) $\alpha$-SMA from old Itch ${ }^{+1+}$ and Itch ${ }^{-1-}$ colon tissues. The data are representative of three or more independent experiments. ${ }^{* \star *} P<0.0001$ based on paired two-tailed Student's $t$-test; the other values are not significant $(P>0.01)$. 
reprobed with anti-Flag. As shown in Supplementary Figure 2a, we found that HIC-5 and Itch were coprecipitated but the deletion of the PPXY motifs in the
HIC-5 protein completely disrupted the Itch-HIC-5 interaction. Furthermore, deletion of all four of the WW domains on Itch (Myc- $\Delta$ Itch) disrupted the Itch-HIC-5 interaction

a Itch-1colon tissue IP : GST-Itch Protein separation

Enzymatic digestion Peptide separation Mass spectrometry
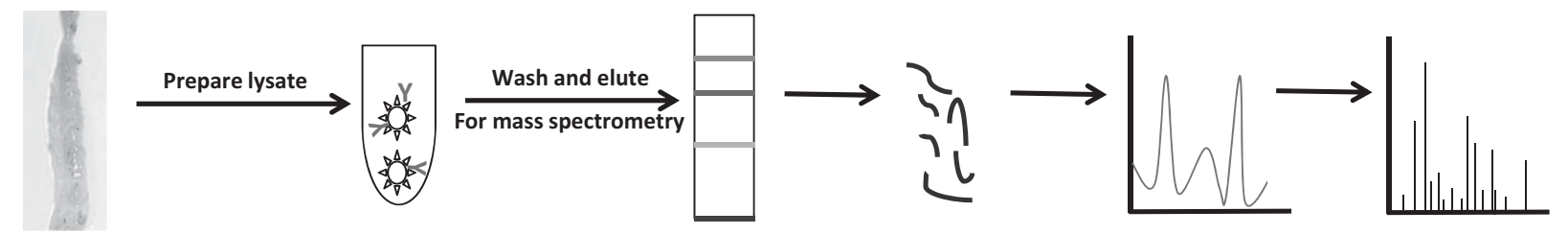

b

\begin{abstract}
Nuclease-sensitive element-binding protein 1 Interferon induced transmembrane protein 3 Mast cell protease 9 Hydrogen peroxide-inducible clone 5 protein Mast cell protease-like protein
\end{abstract}

\begin{abstract}
178 EGQAQQRRPYRRRRFPPYYMRRPYARRPQYSNPPVQGE 214 1 MNHTSQAFITAASGGQPPNYERIKEEYEVAEMGAPHGSAS 40 81 RKRECTQQKIKVEKYILPPNYNVSSKFNDIVLLKLKKQANLT 122 19 SRLGAPKERPPETLTPPPPYGHQPQTGSGESSGTTGDKDH 58 81 RKRECTQQKIKVEKYILPPNYNVSSKFNDIVLLKLKKQANLT 122
\end{abstract}

Figure 2 Itch-interacting proteins can be identified through MS analysis. (a) Schematic presentation of the MS analysis. (b) Proteins containing a PPXY motif identified in MS/MS analysis. A full color version of this figure is available at the Mucosal Immunology journal online.

a

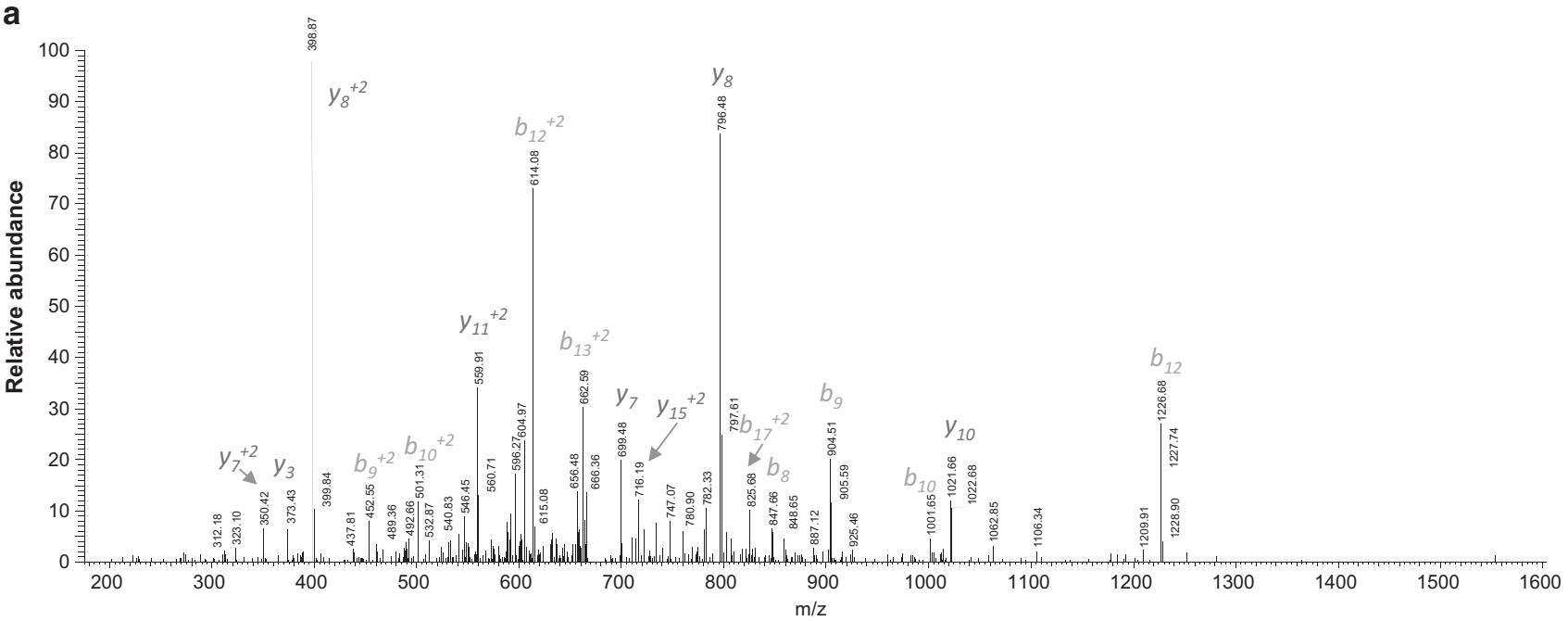

b

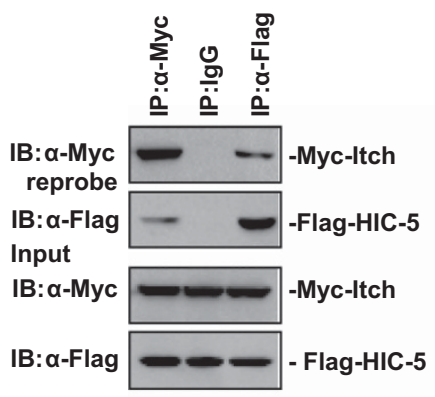

c

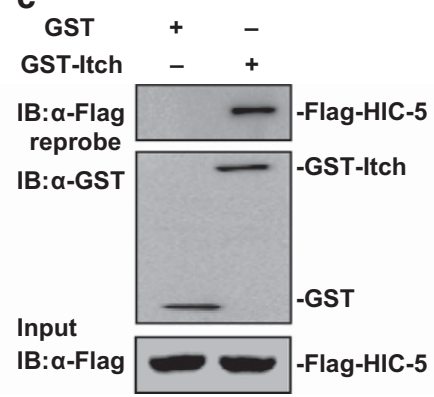

d

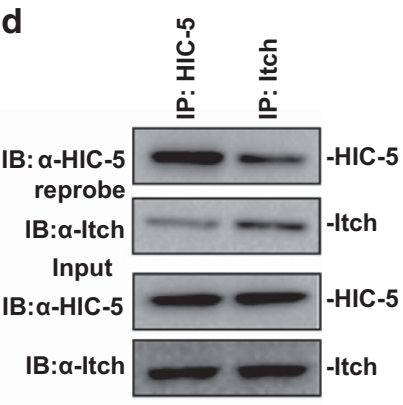

Figure 3 Itch interacts with HIC-5. (a) Itch ${ }^{-1-}$ colon lysate was subjected to pull-down assay with either recombinant GST or GST-Itch. The precipitated proteins were subjected to SDS-PAGE and in-gel digestion. The resulting peptides were analyzed by high-resolution MS/MS. HIC-5 (SwissProt accession No. Q62219) was identified as a specific interactor of Itch protein. An MS/MS spectrum of the peptide 170VQNHLPASGPPQPPAASPTR189 $([\mathrm{M}+\mathrm{H}]+3=674.69 \mathrm{~m} / \mathrm{z})$ belonging to HIC-5 is shown. Observed b- and y-ions are indicated. (b) Immunoassay of lysates of $293 \mathrm{~T}$ cells transfected with plasmids encoding Flag-HIC-5 and Myc-Itch. This was followed by immunoprecipitation (IP) with anti-Myc, anti-Flag, or the control antibody IgG (above blots) and immunoblot analysis (IB) with anti-Myc or anti-Flag (left margin). The Input rows show immunoblot analysis of the samples without immunoprecipitation (throughout). (c) Immunoassay of lysates from 293T cells transfected with Flag-HIC-5 and precipitated with GST or GST-Itch purified from E. coli. Reprobe blot shows GST and GST-Itch, and input shows Flag-HIC-5. (d) Immunoassay of lysates of Itch ${ }^{+/+}$colonic MEFs subjected to immunoprecipitation with anti-HIC-5 or anti-Itch and immunoblot analysis with anti-HIC-5 or anti-Itch. The data are representative of three or more independent experiments. A full color version of this figure is available at the Mucosal Immunology journal online. 
(Supplementary Figure 2b). Taken together, these data suggest that Itch associates with HIC-5 through the 'WW-PPXY' motifs.

\section{Itch targets HIC-5 for K63-linked ubiquitination}

Next, we investigated whether Itch targets HIC-5 for ubiquitination to regulate fibrosis. We transiently transfected $293 \mathrm{~T}$ cells to express Flag-HIC-5, Myc-Itch, and hemagglutinin (HA)-tagged ubiquitin and collected cells $36 \mathrm{~h}$ after transfection. We immunoprecipitated Flag-HIC-5 with anti-Flag and probed the membranes with anti-HA. We observed highmolecular-weight polyubiquitinated forms of HIC-5 that migrated slowly (Figure 4a). This finding suggests that Itch targeted HIC-5 for ubiquitination. To confirm the specificity of Itch as the E3 ligase, we generated an Itch mutant with a substitution of alanine for the cysteine residue at position 830 (Itch-C830A). ${ }^{25}$ Expression of Itch-C830A with WT ubiquitin did not lead to ubiquitination of HIC-5 (Figure 4a).

Since Itch targets its substrates for polyubiquitination via Lys27 (K27)-, K29-, K33-, K48-, and K63-linked ubiquitin chains, ${ }^{26-30}$ we sought to identify the linkage of the Itchmediated ubiquitination of HIC- 5 by using the ubiquitin expression constructs HA-Ub-K48, HA-Ub-K63, HA-Ub-K6, HA-Ub-K0, HA-Ub-K11, HA-Ub-K27, HA-Ub-K29, and HAUb-K33 (in which all of the lysine residues except K48, K63, K6, $\mathrm{K} 0, \mathrm{~K} 11, \mathrm{~K} 27, \mathrm{~K} 29$, and $\mathrm{K} 33$, respectively, are replaced). As shown in Figure $\mathbf{4 b}$ and Supplementary Figure 3, Itch predominantly targeted HIC-5 for K63-linked ubiquitination. To further confirm that Itch targets HIC-5 for K63-linked ubiquitination, we used a ubiquitin mutant with a substitution a

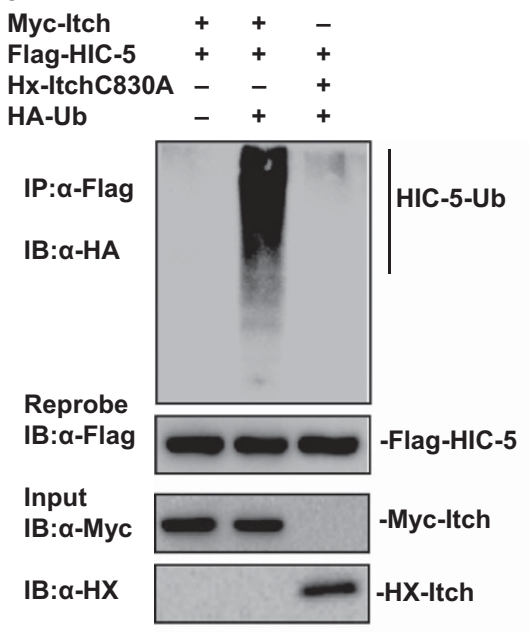

C

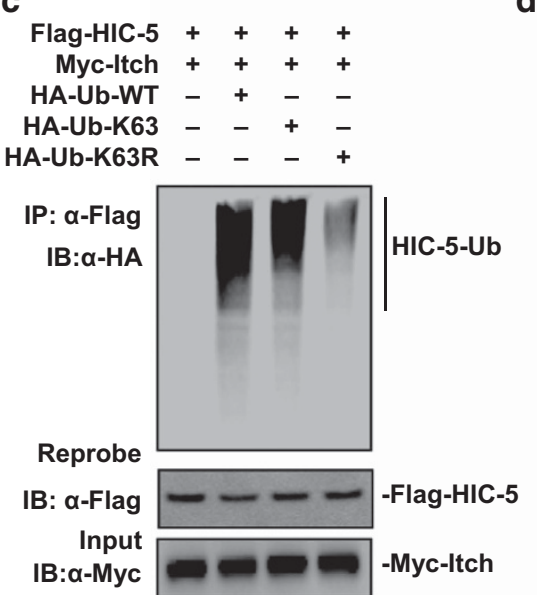

b

d
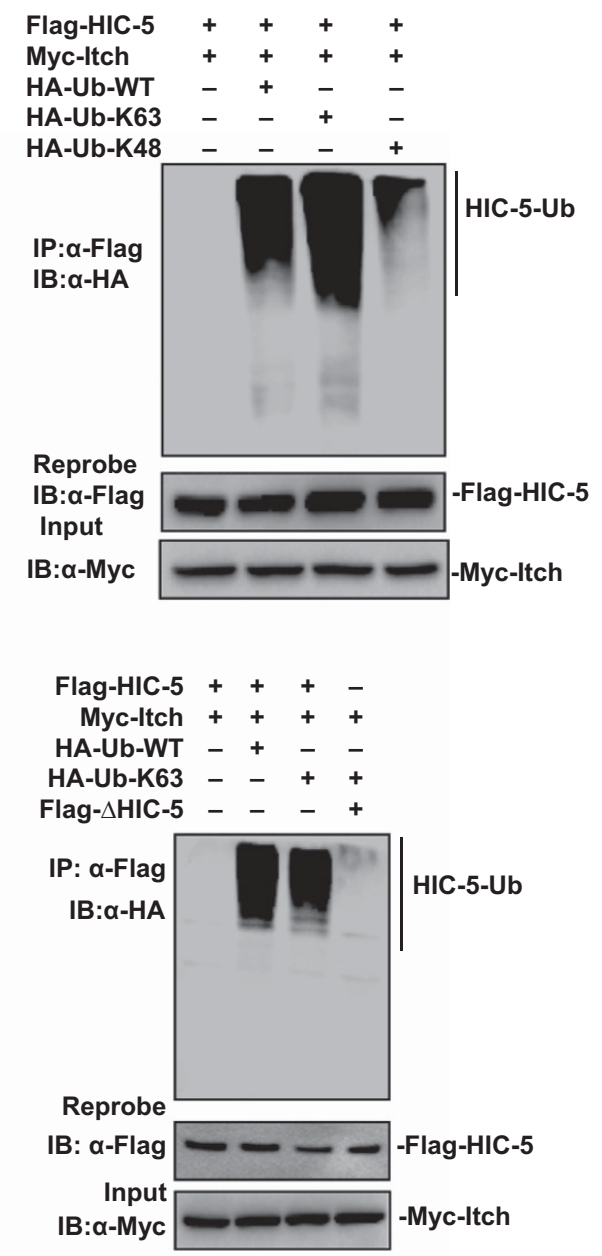

Figure 4 Itch targets HIC-5 via K63-linked ubiquitination. (a) Immunoassay of lysates of 293T cell transfected for $36 \mathrm{~h}$ with various combinations (above lanes) of plasmids encoding Myc-Itch, X-press (HX)-tagged Itch-C830A (HX-ItchC830A), Flag-HIC-5 and HA-tagged WT ubiquitin (HA-Ub WT). This was followed by immunoprecipitation with anti-Flag, immunoblot analysis with anti-HA, and reprobed with anti-Flag. (b) Immunoassay of 293T cells transfected with expression vectors for various combinations (above lanes) of Flag-HIC-5, Myc-ltch, (HA-Ub WT), Ub (K48) and Ub (K63), followed by immunoprecipitation of lysates with anti-Flag and immunoblot analysis with anti-HA. (c) Immunoassay of 293T cells transfected with expression vectors for various combinations (above lanes) of Flag-HIC-5, Myc-Itch, HA-Ub WT, Ub (K63), and Ub (K63R), followed by immunoprecipitation of lysates with anti-Flag and immunoblot analysis with anti-HA. (d) Immunoassay of 293T cells transfected with expression vectors for various combinations (above lanes) of Flag-HIC-5, Myc-ltch, Flag- $\triangle \mathrm{HIC}-5$, HA-Ub WT and Ub (K63), followed by immunoprecipitation of lysates with anti-Flag and immunoblot analysis with anti-HA. The data are representative of three or more independent experiments. 
of arginine for the lysine residue at position 63 (Ub (K63R)). Co-expression of Ub (K63R) and Itch did not ubiquitinate HIC-5 (Figure 4c). To investigate whether the Itch-HIC-5 interaction is required for HIC-5 ubiquitination, we used the Flag- $\Delta$ HIC-5 mutant, which fails to interact with Itch. The Flag- $\Delta$ HIC- 5 mutant was not ubiquitinated by Itch (Figure 4d), which suggests that the Itch-HIC-5 interaction was essential for Itch-mediated ubiquitination of HIC-5.

\section{Itch negatively regulates IL-17-induced HIC-5 in myofibroblasts by lysosomal degradation}

We have recently reported elevated expression of IL-17 in the colonic mucosa of Itch ${ }^{-1-}$ mice. Since IL-17A has been shown to induce fibrosis in different organs by inducing profibrotic gene expression, ${ }^{9,31,32}$ we tested if IL-17 regulates HIC-5 expression. We cultured MEFs from the colon tissues of Itch $^{+1+}$ mice. We then treated the cells with recombinant IL-17 and analyzed the expression of HIC- 5 by real-time PCR and western blot. As shown in Figure 5a and $\mathbf{b}$, IL-17 induced HIC-5 expression in MEFs.

Next, we tested if Itch regulates IL-17-induced HIC-5 in MEFs. We treated Itch ${ }^{+1+}$ and Itch ${ }^{-1-}$ MEFs with IL-17 and analyzed HIC- 5 expression by immunoblotting. As shown in Figure 5c, we observed substantially elevated levels of HIC-5 in Itch $^{-1-}$ MEFs. To investigate the possibility of increased transcription of HIC-5 in Itch $^{-1-}$ MEFs, we performed realtime PCR experiments. However, no significant difference in HIC-5 transcription was observed between WT and Itch $^{-1-}$ cells (Figure 5d). Similar results were obtained when we analyzed HIC- 5 levels following treatment with TGF- $\beta$ (Figure 5e and $\mathbf{f}$ ), which suggests that Itch regulates HIC-5 protein turnover.

Th2-biased differentiation was reported earlier in $\mathrm{Itch}^{-1-}$ T cells. Since IL-13, a Th2 cytokine, is a major factor in colonic fibrosis, ${ }^{33}$ we investigated the potential role of IL-13 in a polysaccharide dextran sodium sulfate (DSS)-induced colitis model in Itch ${ }^{-1-}$ mice. We included 2.5\% DSS in the drinking water of 6- to-10-week-old Itch ${ }^{+/+}$and $\mathrm{Itch}^{-1-}$ mice. On day 8 , we collected colonic mucosa and analyzed the expression of IL-13 by real-time PCR. As shown in Supplementary Figure 4a, DSS treatment induced IL-13 expression in both Itch $^{+1+}$ and Itch ${ }^{-1-}$ mice, and the level of IL-13 was comparable in both sets of colons. Similarly, the expression of Tl1a, another well-documented regulator of colonic inflammation, ${ }^{33}$ was not significantly altered in Itch $^{-1-}$ colonic mucosa in a similar experiment (Supplementary Figure $4 \mathbf{b}$ ). This suggested that IL-13 and Tlla did not play a major role in inflammation and fibrosis in Itch $^{-1-}$ mice.

To investigate the mechanism by which Itch regulates HIC- 5 turnover, we treated WT MEFs with IL-17 and cycloheximide (CHX, a protein synthesis inhibitor), in combination with either chloroquine (a lysosomal inhibitor) or MG132 (a proteasomal inhibitor). As shown in Figure 5g, lanes 1 and 2, CHX treatment resulted in a substantially reduced level of HIC-5. Chloroquine but not MG132 prevented this effect of CHX on HIC- 5 protein level. These results suggest that HIC-5 is degraded via the lysosomal pathway. To test if a defect in the degradation of HIC-5 results in increased abundance of HIC-5 in colonic mucosa of Itch $^{-1-}$ mice, we performed immunoblotting experiments. As shown in Supplementary Figure 5, we found increased HIC-5 in the colons of Itch ${ }^{-1-}$ mice.

\section{Inhibition of HIC-5 in Itch ${ }^{-1-}$ mice decreases fibrotic gene expression}

Next, we tested if inhibition of HIC-5 normalizes the expression of Col I and $\alpha$-SMA by Itch ${ }^{-1-}$ MEFs. We knocked down HIC-5 in Itch $^{-1-}$ MEFs using shRNA (Figure 6a), and the cells were then stimulated with IL-17. As shown in Figure 6b, knocking down HIC- 5 substantially reduced Col I and $\alpha$-SMA expression in the $\mathrm{Itch}^{-1-}$ cells.

To further confirm that Itch-mediated HIC-5 ubiquitination regulates fibrosis, we reconstituted Itch $^{-1-}$ cells with either WT Itch or the Itch-C830A mutant (which lacks the ligase activity) and then stimulated with IL-17. As shown in Figure $\mathbf{6 c}$, reconstitution of WT but not the Itch-C830A mutant inhibited $\mathrm{Col}$ I and $\alpha$-SMA expression. Taken together, these data suggest that Itch regulates the expression of fibrosiscausing genes via ubiquitination of HIC-5.

Finally, we tested if attenuating the IL-17 response in Itch $^{-/-}$ mice prevented colonic fibrosis. For this, we utilized $\mathrm{Itch}^{-1-}$ Rorc $^{-1-}(\mathrm{dKO})$ mice. ${ }^{8}$ As shown in Figure 7a, Itch $^{-1-}$ Rorc $^{-1-}$ $(\mathrm{dKO})$ mice were completely rescued from rectal prolapse and colonic fibrosis compared to Itch $^{-1-}$ mice (Figure $7 \mathbf{b}$ and c). The expression of Col I and $\alpha$-SMA in Itch $^{-1-}$ Rorc $^{-1-}$ mice was significantly reduced compared to that in the $\mathrm{Itch}^{-1-}$ mice (Figure $7 \mathbf{d}$ and $\mathbf{e})$. Collectively, these results suggest that Itch plays an essential role in inhibiting colonic fibrosis.

HIC-5 in the colonic mucosa of ulcerative colitis patients To investigate if a defect in Itch-mediated ubiquitination contributes to fibrosis in UC patients, we analyzed HIC-5 expression by immunoblotting the colonic mucosal tissue of control and UC patient samples. In the majority of the samples that we analyzed, the HIC-5 level was higher in the mucosa of UC patients (Supplementary Figure 6a). Next, we tested if Itch expression is reduced in UC patients and as a result causes the accumulation of HIC-5. Therefore, we reprobed the membranes with anti-Itch antibody. However, no significant change in Itch expression was observed between control and UC patient samples (Supplementary Figure 6a). Next, we investigated if Itch function is defective in UC patients. We immunoprecipitated Itch from both the normal control and UC patient samples, and tested the E3 ligase activity by an in vitro ubiquitination assay. As shown in Supplementary Figure 6b, the ligase activity of Itch protein precipitated from UC patients was substantially reduced, suggesting the possibility of a defect in Itch function in UC patients' mucosa.

\section{DISCUSSION}

Current therapies such as aminosalicylate, steroid and immunomodulator therapies, and anti-TNF- $\alpha$ monoclonal antibodies relieve inflammatory symptoms of IBD. However, 


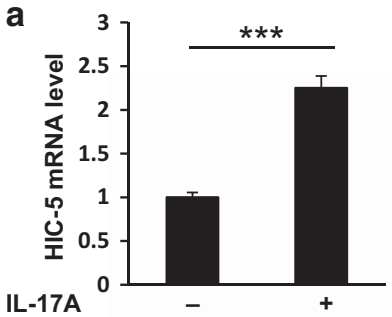

d

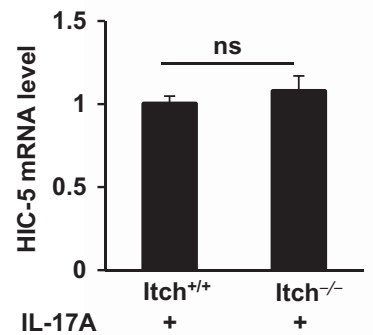

b

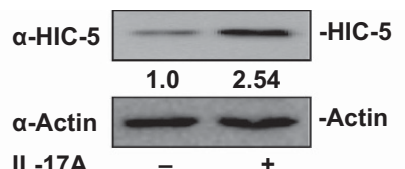

IL-17A

e

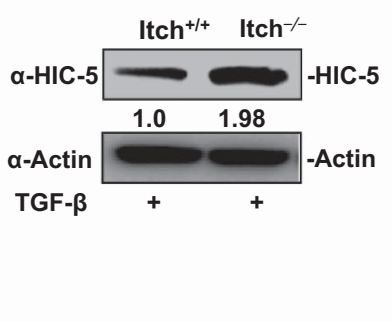

c
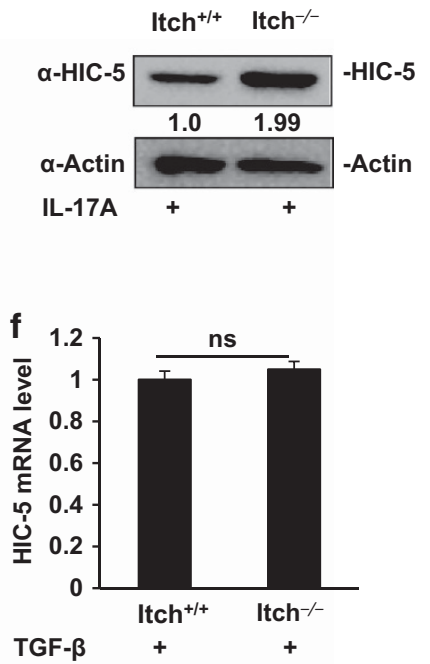

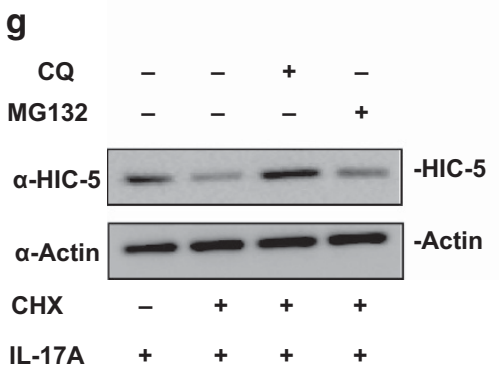

Figure 5 Itch negatively regulates IL-17-induced HIC-5 expression via lysosomal degradation. Itch ${ }^{+/+}$MEFs were either treated with $30 \mathrm{ng} / \mathrm{ml}$ of recombinant murine IL-17A (rlL-17A) or left untreated for $24 \mathrm{~h}$. (a) Relative mRNA level of HIC-5 was measured in Itch ${ }^{+/+} \mathrm{MEFs}$. The relative fold change in the mRNA levels of the gene has been normalized against untreated control of the respective Itch ${ }^{+1+}$ MEFs. (b) Immunoblot analysis of HIC- 5 protein level in lysates of Itch $^{+1+}$ MEFs. Itch ${ }^{+1+}$ and Itch ${ }^{-1}$ MEFs were treated with $30 \mathrm{ng} / \mathrm{ml} \mathrm{of} \mathrm{rlL-17A} \mathrm{for} 24 \mathrm{~h}$. (c) Immunoblot analysis of HIC-5 protein level in lysates of Itch ${ }^{+1+}$ and Itch ${ }^{-1-}$ MEFs after rIL-17A treatment. (d) Relative mRNA level of HIC-5 was measured in Itch ${ }^{+1+}$ and Itch $^{-1-}$ MEFs after rIL$17 \mathrm{~A}$ treatment. Itch ${ }^{+/+}$and Itch $^{-1-}$ MEFs were treated with $10 \mathrm{ng} / \mathrm{ml}$ of recombinant TGF- $\beta$ (rTGF- $\beta$ ) for $24 \mathrm{~h}$. (e) Immunoblot analysis of HIC-5 protein level and (f) relative mRNA level of HIC-5 were measured in lysates of Itch ${ }^{+1+}$ and Itch ${ }^{-1}$ MEFs after rTGF- $\beta$ treatment. The relative fold change in the mRNA levels of the gene has been normalized against the untreated control of the respective ltch ${ }^{+/+} \mathrm{MEFs}$. (g) Itch ${ }^{+/+} \mathrm{MEFs}$ were treated with $30 \mathrm{ng} / \mathrm{ml}$

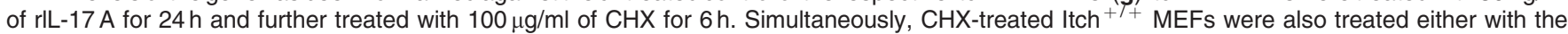
lysosomal inhibitor chloroquine $(50 \mu \mathrm{m})$ or with the proteasome inhibitor MG132 $(5 \mu \mathrm{m})$ for $6 \mathrm{~h}$, and the endogenous protein level of HIC-5 (top) was measured by immunoblot analysis. Untreated Itch ${ }^{+1+}$ MEFs were used as a control. The data are representative of three or more independent experiments. ${ }^{* * \star} P<0.0001$, and the other values are not significant (ns; $>P<0.01$ ) using paired two-tailed Student's $t$-test. For all immunoblot analyses, $\beta$-actin was used as a loading control. Protein levels were normalized to $\beta$-actin and expressed as fold changes (mentioned below the blot) compared with WT controls.

these treatment strategies do not significantly improve fibrotic stricture lesions. ${ }^{34,35}$ Therefore, intestinal fibrosis and its associated complications remain the major causes of surgical intervention in IBD patients. ${ }^{35}$ The molecular mechanisms driving a profibrotic phenotype of the disease remain largely unknown. Currently, no criteria exist to stratify IBD patients into at-risk populations for developing fibrosis. In this report, we have uncovered a novel mechanism by which the E3 ligase Itch inhibits colonic fibrosis by attenuating the expression of profibrotic genes in myofibroblasts, which could be exploited therapeutically to inhibit intestinal fibrosis.

In contrast to the intensive investigation of the immunological mechanisms of intestinal inflammation in IBD, the pathophysiology of fibrosis has remained largely unexplored. Using a mouse model of spontaneous fibrosis, we show that Itch inhibits the expression of profibrotic Col I and $\alpha$-SMA.
Mechanistically, we demonstrate that Itch and HIC-5 interact through their conserved WW motif and PPXY motif, respectively, and that Itch targets HIC-5 for ubiquitination and subsequent proteolysis. Our results are consistent with a report that deletion of HIC-5 attenuated liver fibrosis ${ }^{14}$ and also attenuated myofibroblast differentiation. ${ }^{16}$

HIC-5 (also called TGF- $\beta 111$ ) was originally identified as TGF- $\beta$ and $\mathrm{H}_{2} \mathrm{O}_{2}$-induced gene. ${ }^{17}$ Our results show that HIC-5 expression is also induced by IL-17, suggesting a new role for HIC-5 downstream of IL-17 receptor signaling. IL-17 receptor signals by the recruitment of the adaptor Act1. ${ }^{36}$ Act 1 in turn recruits and ubiquitinates TRAF6, activating downstream $\mathrm{NF}-\kappa \mathrm{B}, \mathrm{CCAAT} / \mathrm{enhancer-binding}$ protein, and the mitogenactivated protein kinase pathway. ${ }^{36}$ It was shown that IL-17 receptor signaling is negatively regulated by deubiquitinases A20 and USP $25 .{ }^{37}$ Our results show that Itch ubiquitinates a 

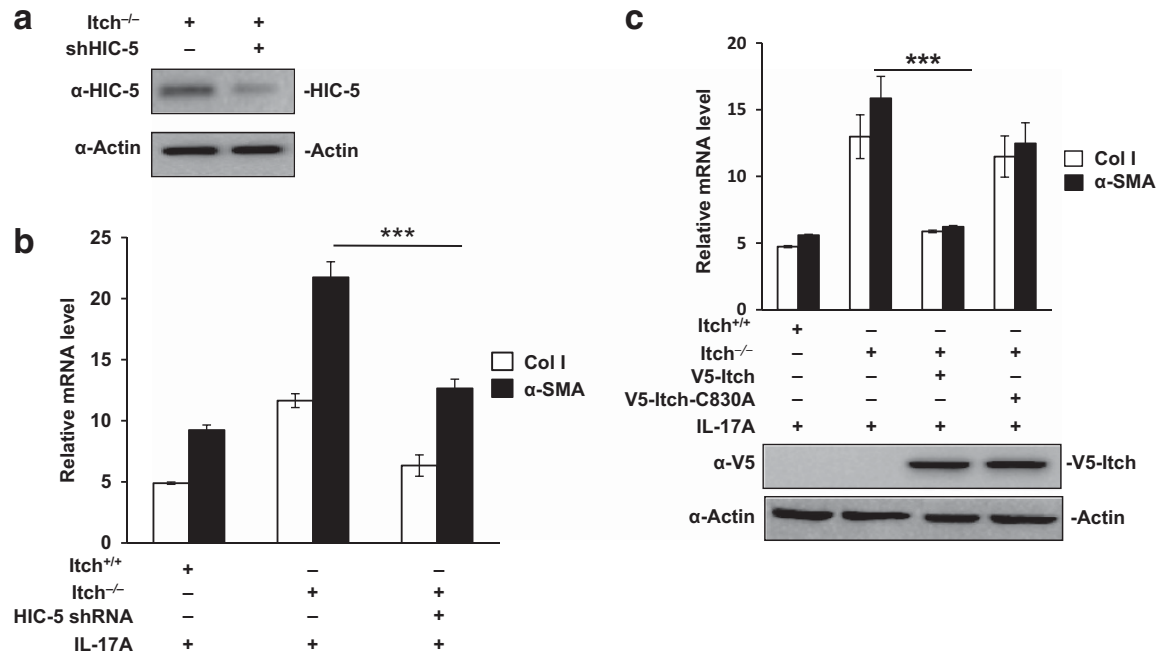

Figure 6 Inhibition of HIC-5 in Itch ${ }^{-1-}$ mice decreases fibrotic gene expression. (a) Itch ${ }^{-1}$ MEFs were treated with HIC-5-specific shRNA (shHIC-5), and immunoblot analysis was done to assess knockdown of HIC-5 in those cells. (b) Real-time PCR analysis of Col I and $\alpha-S M A$ in Itch ${ }^{+/+}$and Itch ${ }^{-1-}$ MEFs treated with shHIC-5 and then treated with $30 \mathrm{ng} / \mathrm{ml}$ of rlL-17A for $24 \mathrm{~h}$. (c) Real-time PCR analysis of Col I and $\alpha-S M A$ in Itch ${ }^{+/+}$and Itch $^{-1-}$ MEFs transduced with lentivirus encoding V5-tagged WT Itch or Itch-C830A and treated with $30 \mathrm{ng} / \mathrm{ml}$ of rlL-17A for $24 \mathrm{~h}$ (top). Immunoblot analysis of lentiviral constructs (bottom). $\beta$-actin was used as a loading control. The relative fold change in the mRNA levels of the genes has been normalized against untreated control of the respective Itch ${ }^{+/+}$MEFs. The data are representative of three or more independent experiments. ${ }^{\star \star \star} P<0.0001$ based on paired two-tailed Student's $t$-test; the other values are not significant $(P>0.01)$.

a

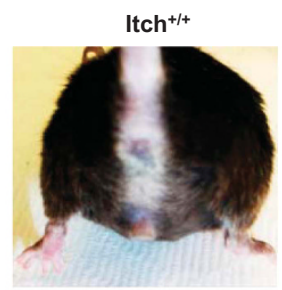

b

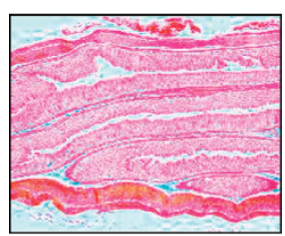

Itch $^{-/-}$

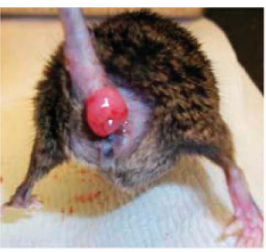

Itch $^{-1-}$

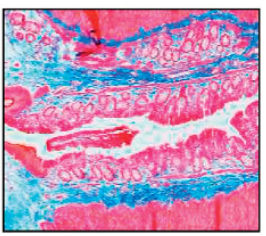

Rorc $^{-1-}$

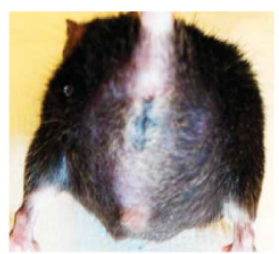

Rorc ${ }^{-/}$

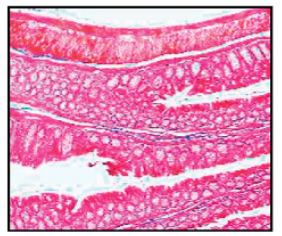

Itch $^{-1-}$ Rorc $^{-1-}$

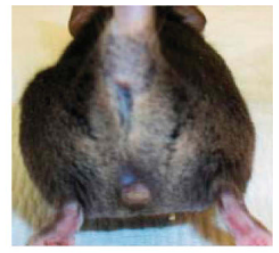

Itch $^{-1-\text { Rorc }^{-1-}}$

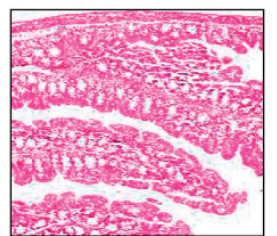

e

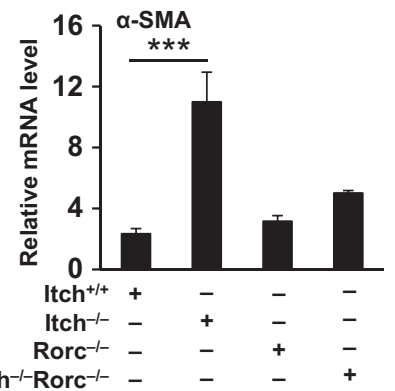

Itch $^{-1-\text { Rorc }^{-1-}}$
C

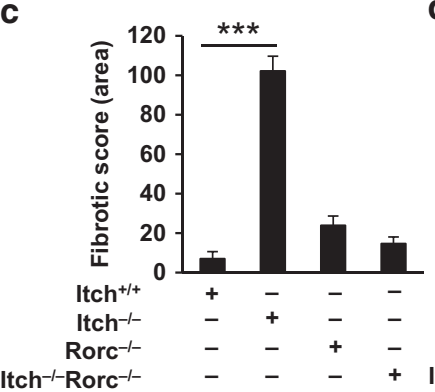

d

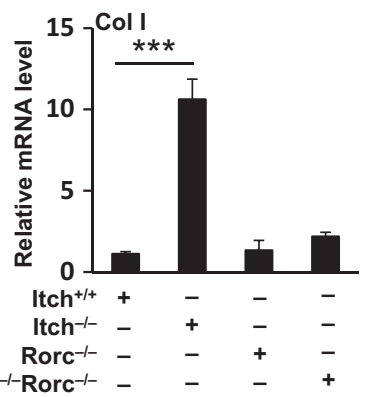

Figure 7 Impeding IL-17 response attenuates colonic fibrosis in Itch ${ }^{-1}$ mice. (a) Representative images of spontaneous rectal prolapse in old Itch $^{+1+}$, Itch ${ }^{-1-}$, Rorc ${ }^{-1-}$, and Itch ${ }^{-1-}$ Rorc $^{-1-}$ mice. (b) Masson trichrome staining of collagen deposition in tissue sections of old Itch ${ }^{+1+}$, Itch ${ }^{-1-}$, Rorc $^{-1-}$, and ltch ${ }^{-1-}$ Rorc $^{-1-}$ mice. (c) Fibrotic scores for sections from part (b). (d,e) Relative mRNA levels of (d) Col I and (e) $\alpha$-SMA from old ltch ${ }^{+/+}$, Itch $^{-1-}$, Rorc ${ }^{-1-}$ and Itch ${ }^{-1-}$ Rorc $^{-1-}$ colon tissue. The relative fold change in the mRNA levels of the genes has been normalized against Itch ${ }^{+1+}$ control colon tissue. The data are representative of three or more independent experiments. ${ }^{\star \star *} P<0.0001$ based on paired two-tailed Student's $t$-test; the other values are not significant $(P>0.01)$. 
downstream effector of IL-17 and negatively regulates IL-17triggered fibrosis. Thus, our findings uncover a novel mechanism by which the IL-17-mediated pathology is inhibited by Itch.

Analysis of Itch expression in the mucosa of UC patients did not show gross change compared to healthy controls. This is consistent with a previous report that Itch expression was normal in UC patients' peripheral blood lymphocytes. ${ }^{38}$ However, we observed that Itch protein isolated from UC patients had reduced ligase activity. The underlying mechanism currently remains unclear. It was demonstrated before that Itch ligase activity is regulated by JNK1-mediated phosphorylation. ${ }^{39}$ Such phosphorylation-dependent regulation of Parkin by the tyrosine kinase c-Abl has been reported in Parkinson disease patients. ${ }^{40}$ Additionally, Itch function was shown to be regulated by the adaptor protein NDFIP1. ${ }^{41}$ Further studies investigating if these forms of regulation are altered in UC patients is required to fully understand the role of Itch in gut inflammation and fibrosis.

In addition to IL-17, Itch also regulates TGF- $\beta$-induced HIC5 expression. Further studies on the physiological function of Itch-mediated regulation of TGF- $\beta$-induced fibrosis are needed. In addition to TGF- $\beta$ and IL-17, additional mediators such as IL-13 and Tlla are critically involved in fibrosis. ${ }^{33}$ However, Itch does not seem to play any significant role in these pathways. Since Itch regulates multiple pathways in hematopoietic and nonhematopoietic cells, we cannot completely exclude the contribution of other cell types and additional mechanisms. Further detailed analysis using tissue/cell-specific conditional deletion of Itch will lead to a more complete understanding of how Itch regulates gut fibrosis. Nevertheless, our studies have uncovered a novel mechanism by which profibrotic gene expression in the colon is inhibited, which could be exploited therapeutically.

\section{METHODS}

Mice. Itch ${ }^{-1-}$ mice were described previously. ${ }^{25}$ Itch $^{-1-}$ Rorc $^{-1-}$ mice were generated by crossing Itch ${ }^{-1}$ with Rorc ${ }^{\text {tm } 1 \text { Litt// }}$ mice. ${ }^{21}$ All mice were housed in micro-isolator cages in the barrier facility of Baylor Institute for Immunology Research. All experiments were performed in accordance with the guidelines of the Institutional Animal Care and Use Committee of Baylor Research Institute.

Human tissue samples. Colonic surgical specimens from patients with UC who were admitted to Baylor University Medical Center for therapeutic bowel resection were collected. This study was approved by the Institutional Review Board of Baylor Research Institute.

Antibodies and reagents. The following antibodies were used in this study: anti-c-Myc (Santa Cruz, Dallas, TX), anti-Flag (Sigma, St. Louis, MO), anti- $\beta$-actin (Sigma), anti-HIC-5 (Santa Cruz), X-press (Invitrogen, San Diego, CA), anti-hemagglutinin (Santa Cruz), antiUb (Santa Cruz), anti-Itch (BD Biosciences, San Jose, CA), GST antibody (Santa Cruz), normal rabbit IgG (Santa Cruz), and normal mouse IgG (Santa Cruz). Recombinant murine IL-17 and recombinant murine TGF- $\beta$ were purchased from PeproTech (Rocky Hill, NJ). HIC-5 shRNA (m) lentiviral particles, control shRNA lentiviral particles-A, polybrene, and puromycin dihydrochloride were purchased from Santa Cruz Biotechnology. Cycloheximide and chloroquine diphosphate were purchased from Sigma.
Cell culture. 293T cells were cultured in DMEM supplemented with $10 \%$ (vol/vol) FBS. Primary mouse colonic MEFs were prepared from 10 -to-12-week-old mice as previously described. ${ }^{42}$

Protein identification by liquid chromatography (LC)-tandem MS. Immunoprecipitated proteins were separated by SDS-PAGE. In-gel digestion with trypsin, followed by protein identification using LC-tandem MS, was performed as described elsewhere. ${ }^{43}$ Briefly, tryptic peptides were resolved on a nano-LC column (Magic AQ C18; Michrom Bioresources, Auburn, CA) and introduced into an Orbitrap mass spectrometer (Thermo Scientific, Waltham, MA). The Orbitrap was set to collect a high-resolution MS1 (FWHM 30,000@400 m/z), followed by the data-dependent collision-induced dissociation spectra on the "top 9" ions in the linear ion trap. Spectra were searched against a human protein database (UniProt release 2011_05) using the X!Tandem/TPP software suite. ${ }^{44}$ Proteins identified with a ProteinProphet probability $\geq 0.9$ false discovery rate $<2 \%$ were considered for further analysis.

Plasmid construction and cell transfection. Myc-Itch, HA-Ub (WT), Ub(K63), Ub(K48), GST-Itch, and HX-Itch (C830A) were described previously. ${ }^{25,26,45}$ Flag-mHIC-5 and Flag-mHIC-5- $\triangle$ PPPPY were created from pMSCV mouse HIC-5 (Addgene, Cambridge, MA) and cloned into pCMV-Tag2B between the BamHI and XhoI sites. Transient transfection of 293T cells was performed using Lipofectamine 2000 (Invitrogen), according to the manufacturer's instructions. HIC-5 shRNA-mediated inhibition in Itch $^{-1-}$ MEFs was done according to the manufacturer's protocol (Santa Cruz Biotechnology).

Real-time PCR analysis. Total RNA was prepared using an RNeasy Mini Kit (Qiagen, Valencia, CA), followed by cDNA synthesis using a Verso cDNA Kit (Thermo Scientific). Quantitative real-time PCR was performed on a Mastercycler EP Realplex (Eppendorf). LightCycler 480 SYBR Green I Master Reaction Mix (Roche, Pleasanton, CA) was used in a $20 \mu \mathrm{l}$ reaction volume. The expression of individual genes was normalized to the expression of actin. Cycling conditions were $95^{\circ} \mathrm{C}$ for $2 \mathrm{~min}$, followed by 50 cycles of $95^{\circ} \mathrm{C}$ for $15 \mathrm{~s}, 55^{\circ} \mathrm{C}$ for $15 \mathrm{~s}$, and $72^{\circ} \mathrm{C}$ for $20 \mathrm{~s}$. The primer sequences for the genes are as follows:

Col I forward primer: 5'-TGCCGTGACCTCAAGATGTG-3'.

Col I reverse primer: $5^{\prime}$-CACAAGCGTGCTGTAGGTGA-3'.

$\alpha$-SMA forward primer: $5^{\prime}$-GTGCTATGTCGCTCTGGACTTTGA-3' $\alpha$-SMA reverse primer: $5^{\prime}$-ATGAAAGATGGCTGGAAGAGGGTC- $3^{\prime}$. HIC-5 forward primer: $5^{\prime}$-GTGCAGGGAATGCCTTGCGC-3'.

HIC-5 reverse primer: 5' -GTGCAGGTGAAGTGGTCTGGATC-3' IL-13 forward primer: $5^{\prime}$-GCAGCATGGTATGGAGTGTG-3'. IL-13 reverse primer: $5^{\prime}$-TGGCGAAACAGTTGCTTTGT- $3^{\prime}$. Tl1a forward primer: $5^{\prime}$-GCTGCCTGTTGTCATTTCC-3'. Tl1a reverse primer: $5^{\prime}$-TCTGGGAGGTGAGTAAACTTG- $3^{\prime}$. $\beta$-Actin forward primer: $5^{\prime}$-GAAATCGTGCGTGACATCAAAG- $3^{\prime}$. $\beta$-Actin reverse primer: $5^{\prime}$-TGTAGTTTCATGGATGCCACAG-3'.

Ubiquitination assay. 293T cells were transfected with Flag-HIC-5 and various constructs, as indicated. MG132 (10 mM) was added $4 \mathrm{~h}$ before cell lysis. Cells were lysed in Nonidet P-40 lysis buffer containing $50 \mathrm{~mm}$ Tris $\mathrm{pH} 8,150 \mathrm{~mm} \mathrm{NaCl}, 1 \% \mathrm{NP}-40$, EDTA-free protease inhibitor cocktail (Roche), $2 \mathrm{~mm}$ sodium fluoride (Boston Bioproducts, Ashland, OR), $10 \mathrm{~mm} \beta$-glycerophosphate (Boston Bioproducts), and $1 \mathrm{~mm}$ sodium orthovanadate (Sigma). Immunoprecipitation was performed using anti-Flag. HIC-5-associated Ub was analyzed by immunoblot using anti-HA, as described previously. ${ }^{25,26,45} \mathrm{An}$ in vitro ubiquitination assay was performed using the E2-Ubiquitin Conjugation Kit (Abcam, Cambridge, MA) with GST-HIC-5 protein as a substrate and Itch immunoprecipitated from either control and UC patients' tissue sample as the E3 ligase according to the manufacturer's protocol. GST-HIC-5 protein was prepared as described previously. ${ }^{25}$

Immunoprecipitation and immunoblot analysis. Cell lysates was prepared from colonic tissues of Itch $^{+/+}$and Itch $^{-/-}$mice after 
homogenizing in NP-40 lysis buffer. Protein estimations were done using the Pierce BCA protein assay kit according to the manufacturer's protocol. Whole-cell lysates were precleared with $20 \mu \mathrm{l}$ of Protein A/G plus agarose beads (Santa Cruz) for $1 \mathrm{~h}$ at $4{ }^{\circ} \mathrm{C}$. Lysates were then incubated with $1 \mu \mathrm{g}$ of the desired antibody overnight at $4{ }^{\circ} \mathrm{C}$ followed by a further $1 \mathrm{~h}$ of incubation at $4{ }^{\circ} \mathrm{C}$ with $25 \mu \mathrm{l}$ of Protein $\mathrm{A} / \mathrm{G}$ beads. The immunocomplexes were washed five times with lysis buffer and denatured using $4 \times$ Laemmli buffer. Further, they were separated by SDS-PAGE and immunoblotted with the indicated antibodies. Blots were visualized using Amersham ECL plus immunoblot analysis detection system (GE, Chicago, IL) on a Biorad ChemiDoc. For reprobing, membranes were stripped by incubation in a stripping buffer (62.5 mm Tris-HCl, pH 6.7; $100 \mathrm{~mm}$ 2-mercaptoethanol and 2\% SDS) at $55^{\circ} \mathrm{C}$ for $45 \mathrm{~min}$ and washed thoroughly before reprobing.

Histology and trichrome staining. Six-micrometer cryosections were fixed for $5 \mathrm{~min}$ in acetone and stained with a Masson trichrome staining kit (Sigma-Aldrich, St. Louis, MO) for detection of collagen deposition. Collagen deposition was quantified on trichrome-stained sections using ImageJ software (NIH, Bethesda, MD) on five fields/ section.

Statistical analysis. The data were analyzed with GraphPad Prism 4 software (La Jolla, CA) to determine statistical significance using paired Student's $t$-test. The data are expressed as mean \pm SD. A $P$-value $<0.05$ was considered significant.

SUPPLEMENTARY MATERIAL is linked to the online version of the paper at http://www.nature.com/mi

\section{ACKNOWLEDGMENTS}

We thank Dr Carson Harrod for critical reading of the manuscript. Acknowledge the contribution of Dr Prasanna K. Santhekadur to this project. This work was supported by grants from the American Cancer Society (122713-RSG-12-260-01-LIB), Cancer Prevention Research Institute of Texas (RP160577), and the Baylor Charles A. Sammons Cancer Center to K. Venuprasad.

\section{AUTHOR CONTRIBUTIONS}

J.P., A.K.S., M.K., T.L.E., and M.Z. performed the experiments, analyzed the data, and assisted in manuscript preparation. V.B. performed MS analysis; A.T. assisted in manuscript preparation; and K.V. conceived the project, designed the experiments, and wrote the manuscript.

\section{DISCLOSURE}

The authors declare no conflict of interest.

c 2018 Society for Mucosal Immunology

\section{REFERENCES}

1. Neurath, M.F. Cytokines in inflammatory bowel disease. Nat. Rev. Immunol. 14, 329-342 (2014).

2. Alvarez, D. et al. A two-hit mechanism for pre-mitotic arrest of cancer cell proliferation by a polyamide-alkylator conjugate. Cell Cycle 5, 1537-1548 (2006).

3. Rieder, F. \& Fiocchi, C. Intestinal fibrosis in IBD-a dynamic, multifactorial process. Nat. Rev. Gastroenterol. Hepatol. 6, 228-235 (2009).

4. Ueno, A., Ghosh, A., Hung, D., Li, J. \& Jijon, H. Th17 plasticity and its changes associated with inflammatory bowel disease. World J. Gastroenterol. 21, 12283-12295 (2015).

5. Catana, C.S., Berindan Neagoe, I., Cozma, V., Magdas, C., Tabaran, F. \& Dumitrascu, D.L. Contribution of the IL-17/L-23 axis to the pathogenesis of inflammatory bowel disease. World J. Gastroenterol. 21, 5823-5830 (2015).

6. Goldberg, R., Prescott, N., Lord, G.M., MacDonald, T.T. \& Powell, N. The unusual suspects-innate lymphoid cells as novel therapeutic targets in IBD. Nat. Rev. Gastroenterol. Hepatol. 12, 271-283 (2015).
7. Korn, T., Bettelli, E., Oukka, M. \& Kuchroo, V.K. IL-17 and Th17 cells. Annu. Rev. Immunol. 27, 485-517 (2009).

8. Zhang, F., Meng, G. \& Strober, W. Interactions among the transcription factors Runx1, RORgammat and Foxp3 regulate the differentiation of interleukin 17-producing T cells. Nat. Immunol. 9, 1297-1306 (2008).

9. Ray, S., De Salvo, C. \& Pizarro, T.T. Central role of IL-17/Th17 immune responses and the gut microbiota in the pathogenesis of intestinal fibrosis. Curr. Opin. Gastroenterol. 30, 531-538 (2014).

10. Shibanuma, M., Mashimo, J., Kuroki, T. \& Nose, K. Characterization of the TGF beta 1 -inducible hic-5 gene that encodes a putative novel zinc finger protein and its possible involvement in cellular senescence. J. Biol. Chem. 269, 26767-26774 (1994).

11. Mashimo, J., Shibanuma, M., Satoh, H., Chida, K. \& Nose, K. Genomic structure and chromosomal mapping of the mouse hic-5 gene that encodes a focal adhesion protein. Gene 249, 99-103 (2000).

12. Yang, L., Guerrero, J., Hong, H., DeFranco, D.B. \& Stallcup, M.R Interaction of the tau2 transcriptional activation domain of glucocorticoid receptor with a novel steroid receptor coactivator, Hic-5, which localizes to both focal adhesions and the nuclear matrix. Mol. Biol. Cell 11, 2007-2018 (2000).

13. Shibanuma, M., Kim-Kaneyama, J.R., Sato, S. \& Nose, K.A. LIM protein, Hic-5, functions as a potential coactivator for Sp1. J. Cell Biochem. 91, 633-645 (2004)

14. Lei, X.F. et al. Hic-5 deficiency attenuates the activation of hepatic stellate cells and liver fibrosis through upregulation of Smad7 in mice. J. Hepatol. 64, 110-117 (2016).

15. Tumbarello, D.A. \& Turner, C.E. Hic-5 contributes to epithelial-mesenchymal transformation through a RhoA/ROCK-dependent pathway. J. Cell Physiol. 211, 736-747 (2007).

16. Varney, S.D. et al. Hic-5 is required for myofibroblast differentiation by regulating mechanically dependent MRTF-A nuclear accumulation. J. Cell Sci. 129, 774-787 (2016)

17. Kim-Kaneyama, J.R., Lei, X.F., Arita, S., Miyauchi, A., Miyazaki, T. \& Miyazaki, A. Hydrogen peroxide-inducible clone 5 (Hic-5) as a potential therapeutic target for vascular and other disorders. J. Atheroscler. Thromb. 19, 601-607 (2012)

18. Hershko, A. \& Ciechanover, A. The ubiquitin system. Annu. Rev. Biochem. 67, 425-479 (1998).

19. Venuprasad, K., Zeng, M., Baughan, S.L. \& Massoumi, R. Multifaceted role of the ubiquitin ligase Itch in immune regulation. Immunol. Cell Biol. 93, 452-460 (2015).

20. Lohr, N.J. et al. Human ITCH E3 ubiquitin ligase deficiency causes syndromic multisystem autoimmune disease. Am. J. Hum. Genet. 86, 447-453 (2010).

21. Kathania, M. et al. Itch inhibits IL-17-mediated colon inflammation and tumorigenesis by ROR-gammat ubiquitination. Nat. Immunol. 17, 9971004 (2016).

22. Dabiri, G., Tumbarello, D.A., Turner, C.E. \& Van de Water, L. TGF-beta1 slows the growth of pathogenic myofibroblasts through a mechanism requiring the focal adhesion protein, Hic-5. J Invest. Dermatol. 128, 280-291 (2008).

23. Inui, S., Shono, F., Noguchi, F., Nakajima, T., Hosokawa, K. \& Itami, S. In vitro and in vivo evidence of pathogenic roles of Hic-5/ARA55 in keloids through Smad pathway and profibrotic transcription. J. Dermatol. Sci. 58, 152-154 (2010).

24. Desai, L.P. et al. Negative regulation of NADPH oxidase 4 by hydrogen peroxide-inducible clone 5 (Hic-5) protein. J. Biol. Chem. 289, 18270-18278 (2014).

25. Ahmed, N. et al. The E3 ligase Itch and deubiquitinase Cyld act together to regulate Tak1 and inflammation. Nat. Immunol. 12, 1176-1183 (2011).

26. Peng, D.J. et al. Noncanonical K27-linked polyubiquitination of TIEG1 regulates Foxp3 expression and tumor growth. J. Immunol. 186, 5638-5647 (2011).

27. Meijer, I.M., van Rotterdam, W., van Zoelen, E.J. \& van Leeuwen, J.E. $\mathrm{Cbl}$ and Itch binding sites in ERBB4 CYT-1 and CYT-2 mediate K48- and K63-polyubiquitination, respectively. Cell. Signal. 25, 470478 (2013).

28. Tao, M., Scacheri, P.C., Marinis, J.M., Harhaj, E.W., Matesic, L.E. \& Abbott, D.W. ITCH K63-ubiquitinates the NOD2 binding protein, RIP2, to influence inflammatory signaling pathways. Curr. Biol. 19, 1255-1263 (2009). 
29. Huang, $H$. et al. K33-linked polyubiquitination of $T$ cell receptor-zeta regulates proteolysis-independent $\mathrm{T}$ cell signaling. Immunity 33 , 60-70 (2010).

30. Chastagner, P., Israel, A. \& Brou, C. Itch/AIP4 mediates Deltex degradation through the formation of K29-linked polyubiquitin chains. EMBO Rep. 7, 1147-1153 (2006).

31. Tan, Z. et al. IL-17 A plays a critical role in the pathogenesis of liver fibrosis through hepatic stellate cell activation. J. Immunol. 191, 1835-1844 (2013).

32. Gasse, P. et al. IL-1 and IL-23 mediate early IL-17 A production in pulmonary inflammation leading to late fibrosis. PloS ONE 6, e23185 (2011).

33. Wallace, K.L., Zheng, L.B., Kanazawa, Y. \& Shih, D.Q. Immunopathology of inflammatory bowel disease. World J. Gastroenterol. 20, 6-21 (2014).

34. Van Assche, G., Geboes, K. \& Rutgeerts, P. Medical therapy for Crohn's disease strictures. Inflamm. Bowel Dis. 10, 55-60 (2004).

35. Wengrower, D. et al. Losartan reduces trinitrobenzene sulphonic acid-induced colorectal fibrosis in rats. Can. J. Gastroenterol. 26, 3339 (2012).

36. Gaffen, S.L., Jain, R., Garg, A.V. \& Cua, D.J. The IL-23-IL-17 immune axis: from mechanisms to therapeutic testing. Nat. Rev. Immunol. 14, 585-600 (2014).

37. Garg, A.V., Ahmed, M., Vallejo, A.N., Ma, A. \& Gaffen, S.L. The deubiquitinase A20 mediates feedback inhibition of interleukin-17 receptor signaling. Sci. Signal. 6, ra44 (2013).
38. Egawa, S. et al. Upregulation of GRAIL is associated with remission of ulcerative colitis. Am. J. Physiol. Gastrointest. Liver Physiol. 295, G163-G169 (2008).

39. Gallagher, E., Gao, M., Liu, Y.C. \& Karin, M. Activation of the E3 ubiquitin ligase Itch through a phosphorylation-induced conformational change. Proc. Natl. Acad. Sci. U S A 103, 1717-1722 (2006).

40. Imam, S.Z. et al. Novel regulation of parkin function through C-Ablmediated tyrosine phosphorylation: implications for Parkinson's disease. J. Neurosci. 31, 157-163 (2011).

41. Oliver, P.M. et al. Ndfip1 protein promotes the function of itch ubiquitin ligase to prevent Tcell activation and Thelper 2 cell-mediated inflammation. Immunity 25, 929-940 (2006).

42. Khalil, H., Nie, W., Edwards, R.A. \& Yoo, J. Isolation of primary myofibroblasts from mouse and human colon tissue. J. Vis. Exp 80, e50611 (2013).

43. Maine, G.N., Li, H., Zaidi, I.W., Basrur, V., Elenitoba-Johnson, K.S. \& Burstein, E. A bimolecular affinity purification method under denaturing conditions for rapid isolation of a ubiquitinated protein for mass spectrometry analysis. Nat. Protoc. 5, 1447-1459 (2010).

44. Nesvizhskii, A.I., Keller, A., Kolker, E. \& Aebersold, R. A statistical model for identifying proteins by tandem mass spectrometry. Anal. Chem. 75, 4646-4658 (2003).

45. Kathania, M., Zeng, M., Yadav, V.N., Moghaddam, S.J., Yang, B. \& Venuprasad, K. Ndfip1 regulates itch ligase activity and airway inflammation via UbcH7. J. Immunol. 194, 2160-2167 (2015). 